\title{
Probing dissociative molecular dications by mapping vibrational wave functions
}

\author{
R. Püttner, ${ }^{1}$ T. Arion, ${ }^{2}$ M. Förstel, ${ }^{2,3}$ T. Lischke, ${ }^{2}$ M. Mucke, ${ }^{2}$ V. Sekushin, ${ }^{1}$ G. Kaindl, ${ }^{1}$ A. M. Bradshaw,,${ }^{2,4}$ and U. Hergenhahn ${ }^{2}$ \\ ${ }^{1}$ Institut für Experimentalphysik, Freie Universität Berlin, Arnimallee 14, D-14195 Berlin-Dahlem, Germany \\ ${ }^{2}$ Max-Planck-Institut für Plasmaphysik, EURATOM Association, Boltzmannstrasse 2, D-85748 Garching, Germany \\ ${ }^{3}$ Max-Planck-Institut für Kernphysik, Saupfercheckweg 1, D-69117 Heidelberg, Germany \\ ${ }^{4}$ Fritz-Haber-Institut der Max-Planck-Gesellschaft, Faradayweg 4-6, D-14195 Berlin, Germany \\ (Received 27 July 2010; revised manuscript received 17 February 2011; published 8 April 2011)
}

\begin{abstract}
We present high-resolution photoelectron-Auger-electron coincidence spectra of methane $\left(\mathrm{CH}_{4}\right)$. Since the vibrational structure in the photoelectron spectrum is resolved, the Auger spectra corresponding to different vibrational levels can be separated. The seven final states of $\mathrm{CH}_{4}^{2+}$ are either dissociative or metastable, but in any case are populated in a repulsive part of their potential-energy curve via the Auger decay. The Auger line shapes can therefore be obtained by mapping the vibrational wave functions of the core-hole state into energy space. We have implemented this connection in the data analysis. By simultaneously fitting the different Auger spectra, detailed information on the energies of the dicationic states and their repulsive potential-energy curves is derived.
\end{abstract}

DOI: 10.1103/PhysRevA.83.043404

PACS number(s): $32.80 . \mathrm{Hd}, 32.80 . \mathrm{Aa}, 33.15 . \mathrm{Ry}$

\section{INTRODUCTION}

Auger spectroscopy is a potentially useful tool for probing the electronic structure of atoms, molecules, and solids. Although information on the valence-band properties of solids is indeed accessible with this technique [1], its main use has been in surface analysis, since the kinetic energies of Auger electrons are element-specific. Less well known is the use of Auger spectroscopy to prepare multiply ionized atoms, molecules, or clusters [2]. In the case of a neutral target species, Auger decay following the ionization of a shallow core level results in the formation of a dication. For atoms, the spectrum consists of sharp lines with a width that is determined by the time scale of the electronic relaxation process, which is of the order of a few femtoseconds. Molecules have additional degrees of freedom due to the nuclear dynamics and normally give rise to more complicated spectra. Already in the primary core ionization, the change in the equilibrium geometry gives rise to the excitation of vibrational substates (see, e.g., [3]). These different initial states for the Auger process lead in turn to different but overlapping spectra of the dicationic species in the final state.

Photoelectron-Auger-electron coincidence spectroscopy can overcome this situation, as was shown originally for metallic copper by Haak et al. [4]. Later, the technique was also applied to problems in molecular Auger decay. In early experiments, coincident detection with zero-kinetic-energy photoelectrons was used to improve the interpretation of Auger spectra [5,6]. Auger spectra from the two nonequivalent $\mathrm{N}$ atoms in $\mathrm{N}_{2} \mathrm{O}$ and from the $\mathrm{S} 2 p_{3 / 2}$ fine-structure component in OCS were also successfully separated $[5,7,8]$. In these experiments, photoelectrons of nonzero kinetic energy were detected. Recently, Bolognesi et al. [9] as well as Ulrich et al. [10,11] have shown for $\mathrm{CO}$ and $\mathrm{O}_{2}$ that photoelectron-Auger-electron coincidence spectroscopy can be used to separate the different vibrational channels in the core-ionized state and to explain the Auger spectra in detail. Reference [11] also includes a more detailed account of photoelectron-Auger-electron coincidence spectroscopy applied to atoms and molecules.
Auger decay of molecules and clusters containing the second-row elements, in particular $\mathrm{C}$ to $\mathrm{Ne}$, usually ends in a state having two vacancies in the outer valence shell. In this dicationic state, the molecule experiences a considerable weakening of the molecular bonds, and possibly also a substantial change of the equilibrium geometry. Although many small molecules are metastable in their lowest dicationic states (see, e.g., [12]), in Auger decay these states often are populated far away from their local energetic minima. This gives rise to broad features in the Auger spectrum, unlike the situation in CO. Other dicationic states may be simply repulsive, leading to the same result. Since the widths of such features are often greater than their separation, the spectrum consists of strongly overlapping peaks, which can only be analyzed qualitatively.

In this paper, we consider the C $1 s$ Auger decay of methane $\left(\mathrm{CH}_{4}\right)$. The dicationic states of this molecule, in particular the ground state, have attracted considerable interest. Methods for their investigation include Auger decay [13,14], charge stripping spectroscopy (see the critical review in [15]), double charge transfer [16-18], photoelectron-ion-ion coincidence spectroscopy [19], photoelectron-photoelectron coincidence spectroscopy [20], coincidence spectroscopy of Auger electrons and ions [21-23], and quantum chemical calculations [15,23-26]. The four lowest dicationic states actually possess a potential-energy surface with a local minimum [23], i.e., $\mathrm{CH}_{4}^{2+}$ can be metastable. In the local minimum, most of these states are planar. At the geometry where the Auger decay takes place, namely at or near the tetrahedral equilibrium geometry of core-ionized $\mathrm{CH}_{4}$, their potential-energy surfaces are above the potential barrier so that the dissociation of methane in the Auger final state is unhindered [21].

We demonstrate in the present paper that in such a situation, a quantitative analysis of high-resolution photoelectronAuger-electron coincidence spectra can still deliver useful information. For a given Auger transition (i.e., for a transition from a vibrational substate of the singly core-ionized molecule into a particular dicationic final state), the vibrational wave function is mapped via the corresponding repulsive 
potential-energy curve onto the spectral line shape in energy space [27]. By simultaneously fitting the Auger spectra for the various vibrational substates and by taking the different line shapes into account, one can obtain detailed information on the electronic final states. Such analyses of doubly ionized cationic states have hitherto not been possible. They demonstrate, however, that the strongly overlapping dicationic final states of $\mathrm{CH}_{4}$ can be separated with the appropriate instrumentation and spectral analysis.

\section{EXPERIMENTAL}

The experiments were carried out on the UE52-SGM beamline of the synchrotron radiation source BESSY II (Berlin, Germany) [28,29]. The beamline was set to deliver vertically linearly polarized radiation. An effusive beam of $\mathrm{CH}_{4}$ was used up to a working pressure of $5 \times 10^{-6} \mathrm{mbar}$. The experimental setup has been described elsewhere in detail $[10,11]$. In brief, photoelectrons are recorded using a set of six time-of-flight spectrometers (TOF) mounted in a plane spanned by the light propagation axis and the electric-field vector of the synchrotron radiation. Each TOF consists of a grounded $4 \mathrm{~mm}$ aperture, a first retarding tube of conical shape, a cylindrical second retarding tube, and a stack of three microchannel plates. Setting suitable retarding voltages, we have obtained a total apparatus energy resolution of $200 \mathrm{meV}$. The transmission varied less than $10 \%$ for photoelectrons within the chosen energy band. Times of flight in the TOF spectrometers were converted to energies by reference measurements of the $\mathrm{CH}_{4}$ C $1 s$ main line excited by the hybrid bunch.

Auger electrons were recorded using a hemispherical electron energy analyzer, which was mounted in the dipole plane under an angle of $54.7^{\circ}$ with respect to the horizontal. The pass energy was set to $300 \mathrm{eV}$, which allows an energy window of about $32 \mathrm{eV}$ to be recorded simultaneously. The Auger-electron energy resolution was better than $1 \mathrm{eV}$; this value is substantially smaller than the widths of the spectral features; see below. For fast, event-based detection of electrons, the analyzer was retrofitted with a delay-line anode (Roentdek) [30]. The electron energies were calibrated to the adiabatic C $1 s$ ionization potential of $I_{p}=290.69(3) \mathrm{eV} \mathrm{[31].}$ About 30000 coincident events, after subtraction of random events, have been recorded in approximately $9 \mathrm{~h}$. The signals in all TOF analyzers were summed. By retarding the electrons in the TOF's, it is possible to record a kinetic-energy interval of some $\mathrm{eV}$, as spanned by typical inner-shell photoelectron bands [3], with vibrational resolution. The detection times in the TOF analyzers (photoelectrons) are referred to the arrival times in the hemispherical analyzer (Auger electrons). This acquisition scheme is therefore independent of the repetition period of the synchrotron radiation [11]. The time dispersion in the hemispheres constitutes the largest contribution to the photoelectron energy uncertainty [30]. The energy resolution achieved nevertheless clearly exceeds that of other schemes for mapping of two-electron events in energy space [11].

\section{RESULTS}

Figure 1 shows the photoelectron-Auger-electron coincidence map (bottom left panel) for $\mathrm{C} 1 s$ photoionization and

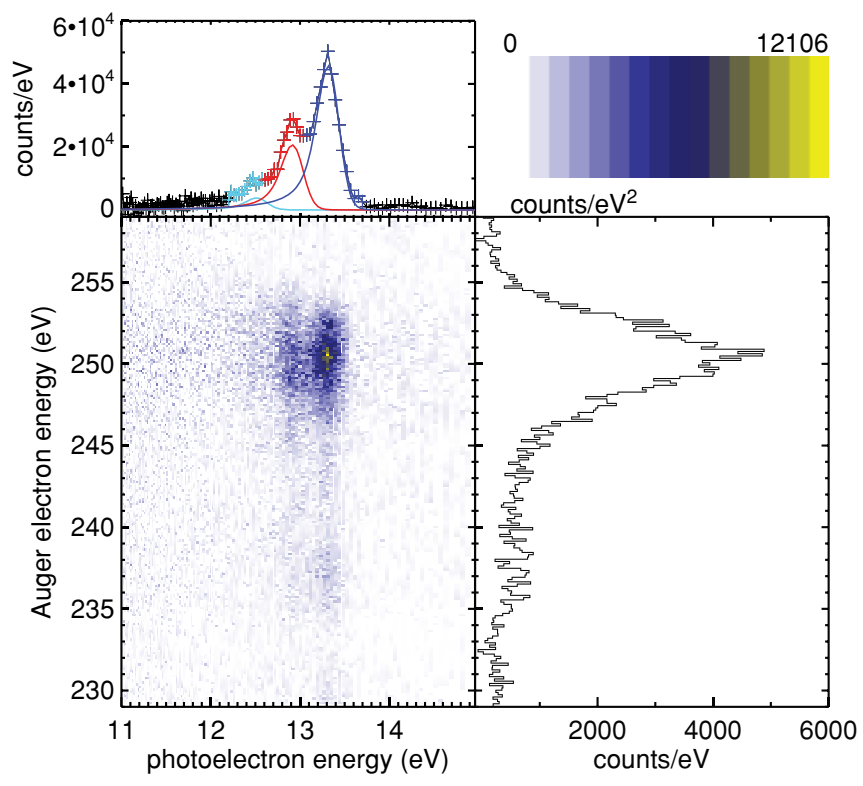

FIG. 1. (Color online) The C $1 s$ photoelectron-Auger-electron coincidence map of $\mathrm{CH}_{4}$ excited by a photon energy of $h v=$ $304 \mathrm{eV}$. The upper panel shows the photoelectron spectrum and the right panel the corresponding Auger spectrum. See text for details.

subsequent $K V V$ Auger decay. Integration along the Auger electron axis results in the photoelectron spectrum (PES), which is shown in the upper panel of the figure and is very similar to previously published high-resolution photoelectron spectra; see, e.g., [32-34]. In this spectrum, the vibrational substates $v=0,1$, and 2 are clearly visible at kinetic energies around $13.3,12.9$, and $12.5 \mathrm{eV}$, respectively. Integration along the photoelectron axis results in the Auger spectrum, which is shown in the right panel of the figure and agrees well with previously published Auger spectra [13].

The two vertical streaks caused by higher count rates at kinetic energies around 13.3 and $12.9 \mathrm{eV}$ represent the Auger spectra originating from the decays starting at the vibrational substates $v=0$ and 1 of the $\mathrm{C} 1 s^{-1}$ core-ionized state. (The remaining intensity in other areas of the panel is the Poisson noise in the coincident electron yield after subtraction of random coincidences.) The blue, red, and cyan data points in the PES of Fig. 1 indicate the intervals used for producing the $v=0,1$, and 2 Auger spectra, respectively. The $v=0$ and 1 spectra are presented in Fig. 2; the $v=2$ has a poor signal-to-noise ratio and is not shown. Although they are dominated by broad structures with typical widths of some $\mathrm{eV}$, indicating dissociative final states, the two spectra show distinct differences. At a kinetic energy of $238 \mathrm{eV}$, for example, the $v=0$ spectrum for Auger decay displays a maximum, while the $v=1$ spectrum possesses a minimum. In addition, the $v=1$ spectrum shows shoulders at kinetic energies of 246 and $253.5 \mathrm{eV}$ that are absent in the $v=0$ spectrum.

\section{A. Modeling of the data}

The observed differences can be understood by the model presented in Fig. 3. The harmonic oscillator in the upper part of the figure represents the potential-energy curve of the $\mathrm{C}$ $1 s^{-1}$ level. The two curves indicated by $v=0$ and 1 represent 


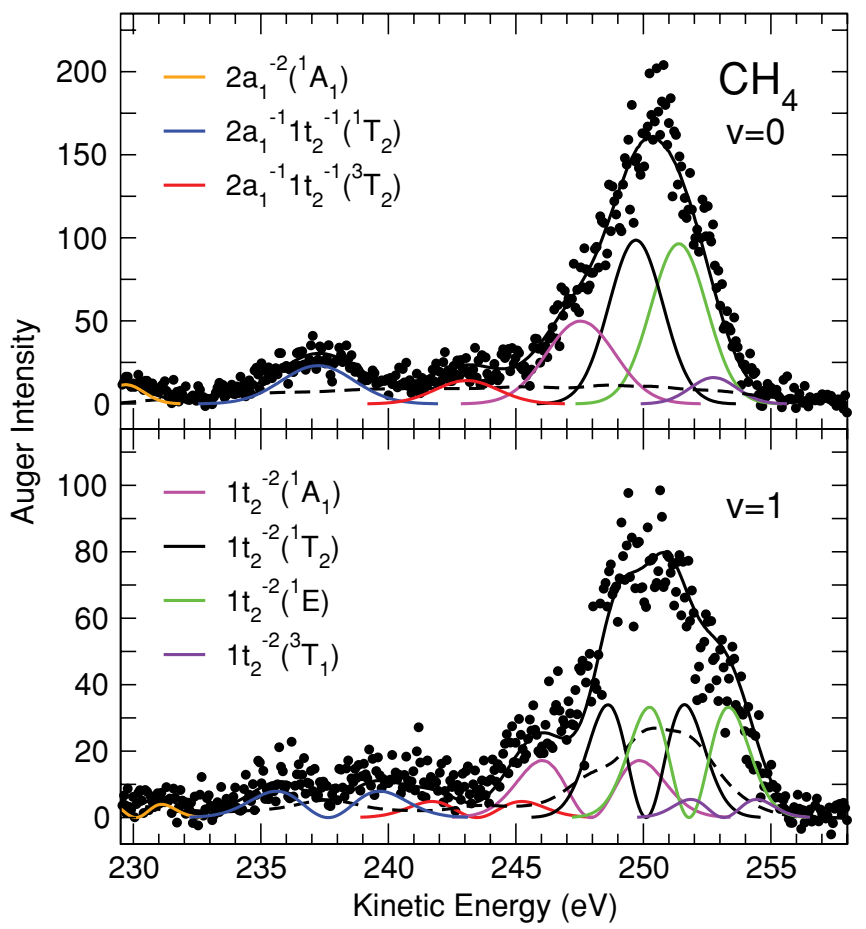

FIG. 2. (Color online) The C $1 s^{-1}$ Auger spectra with $v=0$ (upper panel) and $v=1$ (lower panel) as initial states. The solid lines through the data points represent the fit result. The colored subspectra indicate the contributions of the individual Auger transitions. The assignment is based on a comparison with the calculations of Ortenburger and Bagus [35] and Kvalheim [36].

$\left|\chi_{\text {vib }}(Q)\right|^{2}$ for the two lowest vibrational wave functions $\chi_{\text {vib }}$, with $Q$ being the normal coordinate. The lower curve, with very slight curvature, represents a strongly dissociative

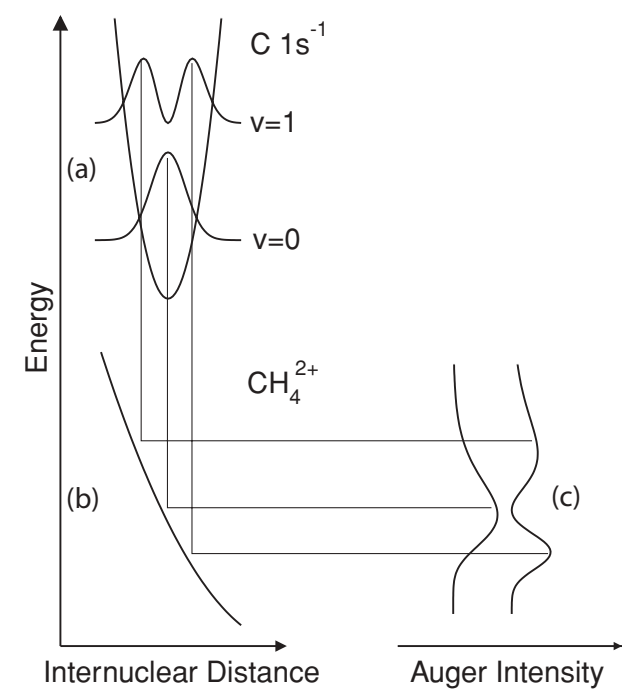

FIG. 3. Schematic picture of the present model. The upper panel (a) indicates a harmonic oscillator representing the potential-energy curve of the bound core ionized state and $\left|\chi_{\text {vib }}(Q)\right|^{2}$ for the two lowest vibrational substates. The lower left panel (b) shows the potentialenergy curve of a dissociative dicationic final state and the right panel (c) the Auger intensities obtained within the Condon reflection approximation. dicationic final state. The continuum wave functions for the nuclear motion in the dicationic final states possess a maximum close to the wall defined by the potential-energy curve and show practically no differences in their nodal structure. As a result, the wave functions can be approximated by a $\delta$ function at the turning point [37]. This is known as the Condon reflection approximation and results in an energy-dependent Auger intensity, which mirrors the vibrational wave function of the core-ionized state, as shown schematically in Fig. 3.

Removing two electrons from the outer valence shell of methane is expected to result in three different configurations, namely $2 a_{1}^{-2}, 2 a_{1}^{-1} 1 t_{2}^{-1}$, and $1 t_{2}^{-2}$. It is known from theory that these can result in seven electronic states: $2 a_{1}^{-2}\left({ }^{1} A_{1}\right)$, $2 a_{1}^{-1} 1 t_{2}^{-1}\left({ }^{1} T_{2},{ }^{3} T_{2}\right)$, and $1 t_{2}^{-2}\left({ }^{1} A_{1},{ }^{1} T_{2},{ }^{1} E,{ }^{3} T_{1}\right)$, in order of decreasing binding energy of the state (corresponding to increasing Auger electron energy) [35]. The first final dicationic configuration is related to the Auger peak at $\cong 230 \mathrm{eV}$ in the spectrum presented in Fig. 1, the second configuration to the peaks at $\cong 238$ and $\cong 243 \mathrm{eV}$, and the last group of states to the broad peak at $\cong 250 \mathrm{eV}$. Two calculations predict additional three-hole, one-particle states in the energy region of the $2 a_{1}^{-2}\left({ }^{1} A_{1}\right)$ [25,36], but these are hardly relevant for the present study (see below).

For a detailed understanding of the spectra presented in Fig. 2, and to extract information on the dicationic states themselves, we have implemented the model described above in a fit analysis, where both spectra have been fitted simultaneously. By assuming a harmonic-oscillator potential for the core-ionized state, the probability density for the vibrational ground state of the symmetric stretching mode, $\left|\chi_{\mathrm{vib}}\left(R-R_{e}\right)\right|^{2}$, is given by

$$
\left|\chi_{\mathrm{vib}}\left(R-R_{e}\right)\right|^{2}=\frac{a}{\sqrt{\pi}} e^{-a^{2}\left(R-R_{e}\right)^{2}},
$$

with $R_{e}$ being the $\mathrm{C}-\mathrm{H}$ equilibrium distance and $a^{2}=\frac{4 \mu \omega}{\hbar}$. Here, $\mu$ is the mass of the hydrogen atom and $\omega$ is the frequency of the symmetric stretching mode. The factor 4, which is absent in the case of a diatomic molecule, is due to four equivalent bond distances in the symmetric stretching mode. Using a straight line for the final-state potential-energy curve, we obtain

$$
I(E) \propto \frac{1}{\sqrt{\pi} \sigma} \mathrm{e}^{-\left(\frac{E-E_{0}}{\sigma}\right)^{2}}
$$

for the line shapes of the Auger transitions to the dissociative dicationic states in the $v=0$ spectrum. Here, $E_{0}$ is the energy difference between the $\mathrm{C} 1 s^{-1}(v=0)$ level and the potential energy of the strongly dissociative state at the equilibrium distance of the core-ionized state. The quantity $\sigma$ represents the widths of the line profiles in the spectra. By comparing the exponents of the two exponential functions, one obtains for the slope of the potential-energy curve

$$
X_{\text {slope }}=\frac{E-E_{0}}{R-R_{e}}=a \sigma .
$$

In an analogous way, we derive from

$$
\left|\chi_{\mathrm{vib}}\left(R-R_{e}\right)\right|^{2}=\frac{2 a^{3}}{\sqrt{\pi}}\left(R-R_{e}\right)^{2} e^{-a^{2}\left(R-R_{e}\right)^{2}}
$$


TABLE I. Summary of the results from our fit analysis, together with a comparison of the relative Auger intensities. Here, $E_{\text {kin }}, X_{\text {slope }}$, and $I_{\text {expt }}$ are the results for the experimental Auger kinetic energy, the slope of the final-state potential-energy curve [Eq. (1)], and the intensity normalized to the $\mathrm{C} 1 s^{-1} \rightarrow 2 a_{1}^{-1} 1 t_{2}^{-1}\left({ }^{1} T_{2}\right)$ transition, which is well-separated and provides the smallest error bars, respectively. All errors are given in parentheses and apply to the last digit(s). Our data for $E_{\text {kin }}$ are additionally subject to an error of $0.1 \mathrm{eV}$ due to calibration of the energy scale, which has not been included in the error bars. $I_{\text {th }}$ are the relative Auger intensities as calculated by various approaches.

\begin{tabular}{|c|c|c|c|c|c|c|}
\hline \multirow[b]{2}{*}{ Final state } & \multirow{2}{*}{$\begin{array}{l}E_{\text {kin }} \\
(\mathrm{eV})\end{array}$} & \multirow{2}{*}{$\begin{array}{c}X_{\text {slope }} \\
(\mathrm{eV} / \AA \AA)\end{array}$} & \multicolumn{4}{|c|}{ Relative intensity } \\
\hline & & & $I_{\text {expt }}$ & $I_{\mathrm{th}}(\mathrm{RHF})^{\mathrm{a}}$ & $I_{\mathrm{th}}(\mathrm{CI})^{\mathrm{a}}$ & $I_{\mathrm{th}}^{\mathrm{b}}$ \\
\hline $1 t_{2}^{-2}\left({ }^{3} T_{1}\right)$ & $252.75(91)$ & $-25.4(9.7)$ & $0.44(75)$ & & & 0.00 \\
\hline $1 t_{2}^{-2}\left({ }^{1} E\right)$ & $251.39(35)$ & $-30.4(1.0)$ & $3.21(49)$ & 1.00 & 2.62 & 0.00 \\
\hline $1 t_{2}^{-2}\left({ }^{1} T_{2}\right)$ & 249.71(17) & $-29.5(0.9)$ & $3.18(68)$ & 1.50 & 5.23 & 2.24 \\
\hline $1 t_{2}^{-2}\left({ }^{1} A_{1}\right)$ & $247.54(11)$ & $-37.5(1.6)$ & $2.04(28)$ & 0.20 & 1.05 & 0.06 \\
\hline $2 a_{1}^{-1} 1 t_{2}^{-1}\left({ }^{3} T_{2}\right)$ & $243.07(14)$ & $-34.5(3.1)$ & $0.53(8)$ & 0.30 & 0.74 & 0.27 \\
\hline $2 a_{1}^{-1} 1 t_{2}^{-1}\left({ }^{1} T_{2}\right)$ & 237.24(8) & $-39.7(1.4)$ & 1 & 1 & 1 & 1 \\
\hline $1 t_{2}^{-3} 2 t_{2}\left({ }^{3} T_{2}\right)$ & & & & & 0.02 & \\
\hline $1 t_{2}^{-3} 3 a_{1}\left({ }^{1} T_{2}\right)$ & & & & & 0.16 & \\
\hline $2 a_{1}^{-2}\left({ }^{1} A_{1}\right)$ & $229.70(14)$ & $-19.9(2.1)$ & $0.25(6)$ & 0.47 & 0.50 & 0.75 \\
\hline
\end{tabular}

${ }^{\mathrm{a}}$ Reference [36], average values for two different sets of radial wave functions.

${ }^{\mathrm{b}}$ Reference [38].

for the first excited vibrational substate

$$
I(E) \propto \frac{2\left(E-E_{0}-\hbar \omega\right)^{2}}{\sqrt{\pi} \sigma^{3}} \mathrm{e}^{-\left(\frac{E-E_{0}-\hbar \omega}{\sigma}\right)^{2}}
$$

for the line shapes of the Auger transitions in the $v=1$ spectrum. Note that for a given transition, $\sigma$ is identical in the $v=0$ and 1 spectra. Besides identical values for $\sigma=X_{\text {slope }} / a$, the simultaneous fitting of both spectra also allows the same value for $E_{0}$ to be used for a given Auger transition. In the $v=1$ spectrum, the line shapes are shifted by the vibrational energy of the core-ionized state, $\hbar \omega$, to higher energies to account for energy conservation during ionization and the subsequent Auger process. Similarly, the relative intensities of the different Auger transitions were kept identical in the $v=0$ and 1 spectra. In this way, the fit to the data in Fig. 2 was performed with three parameters (energy position, width, and intensity) for each of the Auger transitions. A background subtraction involving two parameters was performed for each spectrum. In addition, $v=0$ Auger decays contribute to the $v=1$ spectrum and vice versa. These contributions are

TABLE II. Summary of the final-state energies obtained from the fit analysis for the seven Auger transitions. Given are the absolute energy positions $E_{\text {expt }}$ as available from several techniques and theory [ $E_{\text {expt }}(\mathrm{Au})$ : this work], and energy differences $\left(\Delta E_{\text {expt }}, \Delta E_{\text {th }}\right)$ relative to the isolated $2 a_{1}^{-1} 1 t_{2}^{-1}\left({ }^{1} T_{2}\right)$ state. The latter enables our results to be compared to the authors who have not put their results on an absolute scale. All values are given in $\mathrm{eV}$ and the errors given in parentheses apply to the last digit(s). The error bars on $E_{\text {expt }}(\mathrm{Au})$ include statistical errors from our fit as given in Table I, as well as additional scaling errors of $0.1 \mathrm{eV}$ for the calibration of the energy scale and $0.03 \mathrm{eV}$ for the reference value of the $\mathrm{C} 1 s$ ionization energy [31].

\begin{tabular}{|c|c|c|c|c|c|c|c|c|c|c|}
\hline Final state & $E_{\text {expt }}(\mathrm{Au})^{\mathrm{i}}$ & $E_{\text {expt }}(\mathrm{DCT})^{\mathrm{a}}$ & $E_{\text {expt }}(\mathrm{DCT})^{\mathrm{b}}$ & $E_{\mathrm{th}}{ }^{\mathrm{c}}$ & $E_{\mathrm{th}}{ }^{\mathrm{d}}$ & $E_{\mathrm{th}}{ }^{\mathrm{e}}$ & $\Delta E_{\text {expt }}(\mathrm{Au})^{\mathrm{i}}$ & $\Delta E_{\mathrm{th}}^{\mathrm{f}}$ & $\Delta E_{\mathrm{th}^{\mathrm{g}}}^{\mathrm{g}}$ & $\Delta E_{\text {expt }}(i, e)^{\mathrm{h}}$ \\
\hline $1 t_{2}^{-2}\left({ }^{3} T_{1}\right)$ & $37.94(1.01)$ & $38.3(4)$ & $38.2(4)$ & 37.13 & 36.91 & 38.7 & $15.51(99)$ & & 15.31 & \\
\hline $1 t_{2}^{-2}\left({ }^{1} E\right)$ & $39.30(45)$ & $38.6(4)$ & $39.2(4)$ & 38.17 & 37.67 & 39.7 & $14.15(43)$ & 13.91 & 14.65 & 13.6 \\
\hline $1 t_{2}^{-2}\left({ }^{1} T_{2}\right)$ & $40.98(27)$ & $40.2(4)$ & $40.5(4)$ & 40.11 & 38.99 & 41.0 & $12.47(25)$ & 12.37 & 12.95 & 12.0 \\
\hline $1 t_{2}^{-2}\left({ }^{1} A_{1}\right)$ & $43.15(21)$ & $42.3(4)$ & $42.1(1.0)$ & 42.57 & 40.85 & 42.4 & $10.30(19)$ & 10.00 & 10.89 & 9.6 \\
\hline $2 a_{1}^{-1} 1 t_{2}^{-1}\left({ }^{3} T_{2}\right)$ & $47.62(24)$ & & $46.7(8)$ & 46.86 & 45.73 & 48.3 & $5.83(22)$ & 6.46 & 6.20 & 7.0 \\
\hline $2 a_{1}^{-1} 1 t_{2}^{-1}\left({ }^{1} T_{2}\right)$ & $53.45(18)$ & & $51.0(1.0)$ & 53.19 & 51.39 & 54.8 & 0 & 0 & 0 & 0 \\
\hline $2 a_{1}^{-2}\left({ }^{1} A_{1}\right)$ & $60.99(24)$ & & & 61.4 & 59.08 & 62.4 & $-7.54(22)$ & -7.64 & -7.99 & -8.4 \\
\hline
\end{tabular}

${ }^{\mathrm{a}}$ Reference [17], double charge transfer.

${ }^{\mathrm{b}}$ Reference [18], double charge transfer.

${ }^{\mathrm{c}}$ Reference [35], theory (SCF).

${ }^{\mathrm{d}}$ Reference [39], theory [ADC(2)].

${ }^{\mathrm{e}}$ Reference [23], theory (CAS).

${ }^{\mathrm{f}}$ Reference [36], theory (CI calculations).

${ }^{\mathrm{g}}$ Reference [25], theory (VB).

${ }^{\mathrm{h}}$ Reference [22], fragment ion, Auger-electron coincidence measurement $(i, e)$.

${ }^{\mathrm{i}}$ This work. 
represented by the dashed black subspectrum in Fig. 2. They can be understood from the blue, red, and cyan subspectra in the PES of Fig. 1, which represent the fit results for the vibrational substates $v=0,1$, and 2 , respectively. These lines overlap strongly and, in particular, it can be seen that the post-collision interaction tail of the $v=0$ state (blue subspectrum) contributes significantly to the interval used to form the $v=1$ spectrum (red data points). We have taken this into account by fitting each Auger spectrum in Fig. 2 with contributions of transitions starting from both the $v=0$ and the $v=1$ sublevel; these contributions were weighted in the individual spectra by the relative intensity of these states in the respective interval of photoelectron energies, as given by the high-resolution PES (see top panel in Fig. 1). As a cross-check, we have determined the $\mathrm{C} 1 s^{-1}(v=1)$ to $\mathrm{C}$ $1 s^{-1}(v=0)$ intensity ratio to be 0.467 , derived from the fit shown in Fig. 1. This ratio is in good agreement with previous studies $[3,32,33]$.

\section{B. Fit results}

The results of the fit analysis are represented by the solid lines in Fig. 2, with the colored subspectra indicating the contributions to the individual Auger transitions. Assignment of the lines to the different dicationic states follows the literature (see below). The good agreement between the data points and the fit shows that the differences in the $v=0$ and the $v=1$ spectrum can be explained by the different line shapes that originate from the $\left|\chi_{\text {vib }}(Q)\right|^{2}$ distribution of the vibrational substates in the core-ionized state. The transition at $\cong 238 \mathrm{eV}$ illustrates particularly well the model shown in Fig. 3: the double-peak structure of the $v=1$ spectrum clearly corresponds to the single peak in the $v=0$ spectrum. Such a correspondence is also present in the broad peak around $250 \mathrm{eV}$, but the details are not resolved due to the presence of five strongly overlapping Auger transitions. Only the shoulders at $\cong 246$ and $\cong 253.5 \mathrm{eV}$ in the $v=1$ spectrum, which originate from transitions to the final states $1 t_{2}^{-2}\left({ }^{1} A_{1}\right)$ and $1 t_{2}^{-2}\left({ }^{1} E\right)$, respectively, are indications for the underlying line shapes. For the present fit analysis these shoulders are, however, sufficient for a good estimate of all contributions of the above-mentioned transitions to the $v=0$ and 1 Auger spectra.

Our fit results are given in Table I, together with theoretical results of Kvalheim [36], who performed calculations both in the restricted Hartree-Fock (RHF) approximation as well as on the configuration-interaction (CI) level, and Higashi et al. [38] for the relative intensities of the Auger lines. In the RHF approximation of Kvalheim, the relative intensities of the Auger transitions to the final states $1 t_{2}^{-2}\left({ }^{1} E\right), 1 t_{2}^{-2}\left({ }^{1} T_{2}\right)$, $1 t_{2}^{-2}\left({ }^{1} A_{1}\right)$, and $2 a_{1}^{-1} 1 t_{2}^{-1}\left({ }^{3} T_{2}\right)$ are too low compared to the experimental results. His CI calculations are better at reproducing the experimental intensity ratios, although there are still considerable discrepancies between the experimental and the theoretical results. The Auger transitions to the satellite final states, $1 t_{2}^{-3} 2 t_{2}\left({ }^{3} T_{2}\right)$ and $1 t_{2}^{-3} 3 a_{1}\left({ }^{1} T_{2}\right)$, predicted by Kvalheim [36] in the CI calculations, are not observed in the present experimental spectra. However, their predicted intensities are much lower than for the states observed, so that the statistics of the present spectra do not rule out the existence of these transitions. For the Auger transition to the dicationic ground state, $1 t_{2}^{-2}\left({ }^{3} T_{1}\right)$, Kvalheim did not calculate the Auger intensity. However, the Auger intensities due to the triplet states are expected to be lower than those due to the corresponding singlet states since the triplet states require a spin flip, so that the experimentally obtained intensity of this state is reasonable. The large experimental error bar for this intensity includes a possibility of $\cong 25 \%$ that the corresponding transition is absent in the Auger spectrum. The calculations of Ref. [38] actually predict a vanishing intensity for the $1 t_{2}^{-2}\left({ }^{3} T_{1}\right)$ state, however the agreement between the predicted and the measured intensities of the other well-resolved states is not satisfactory.

From the Auger transition energies given in Table I, the potential energy of the corresponding final states at the geometry of the Auger decay can be calculated from $E_{\text {expt }}=I_{p}-E_{\text {kin. }}$. For this purpose, the value of the C $1 s$ binding energy of Myrseth et al. [31] has been used. Our results for the energies of the states are summarized in Table II, and in graphical form in Fig. 4. The statistical error bars are also given. Systematic errors caused by effects beyond the fit model (see above) have not been investigated, since a reduced $\chi^{2}$ of $\cong 1.25$ indicates that the statistics represents the limiting factor. For comparison, we have included theoretical

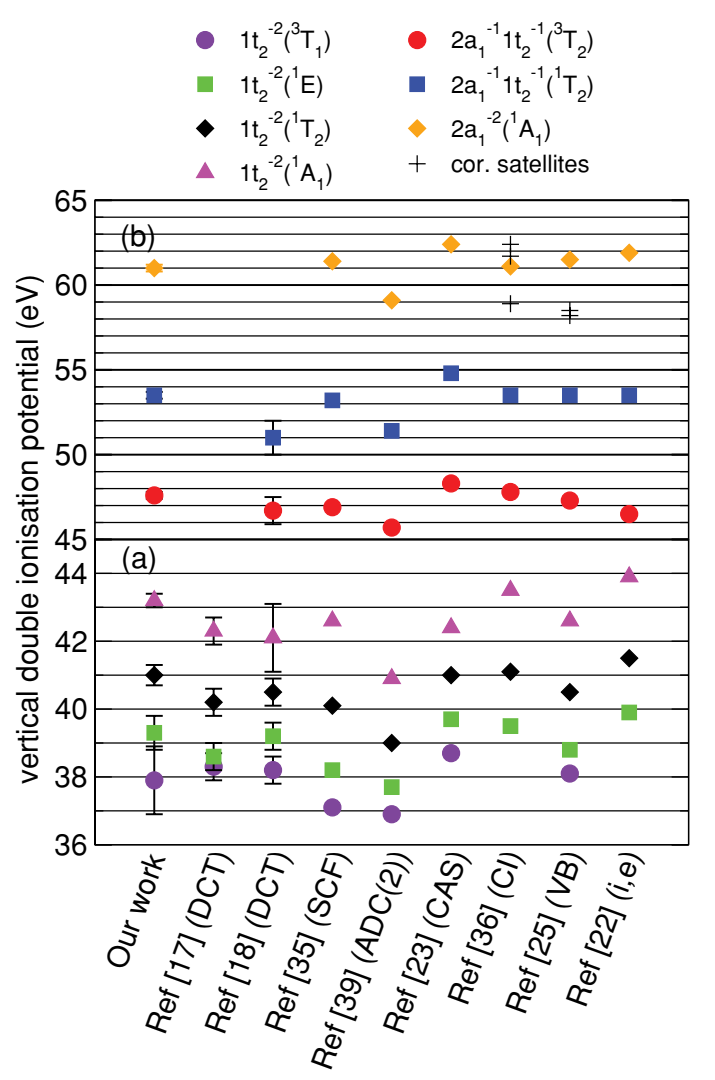

FIG. 4. (Color online) Final-state energies for the $\mathrm{CH}_{4}^{2+}$ dicationic states according to different authors. Panel (a) shows the four states below the most intense Auger line, panel (b) shows the remaining states on a different energy scale. See the description of Table II for details. Relative energies (the three rightmost data sets) have been aligned to our value for the $2 a_{1}^{-1} 1 t_{2}^{-1}\left({ }^{1} T_{2}\right)$ state. Error bars are explained in the caption of Table II. 
results reported by various authors. The CI calculations of Kvalheim [36] agree for all states except $1 t_{2}^{-2}\left({ }^{1} A_{1}\right)$ with the experimental results within the error bars; for the final state $1 t_{2}^{-2}\left({ }^{1} A_{1}\right)$, the deviation is only slightly larger than the error bars. This agreement clearly supports the present fit analysis and justifies our assignment of the observed Auger transitions.

\section{DISCUSSION}

The energies of the doubly ionized states of methane have been discussed a number of times in the literature. After several experiments had given rise to divergent results, it was recognized that different techniques probe the doubly ionized states at different parts of their potential-energy curves (see e.g., [15]). The internal excitation energy of the first four (metastable) states of $\mathrm{CH}_{4}^{2+}$ following a vertical transition from the ground state has been calculated by Flammini et al. [23] to be between 3.9 and $8.6 \mathrm{eV}$. This corresponds to the difference in $E_{\text {expt }}$ between a vertical and a (hypothetical) adiabatic double-ionization experiment.

In this context, it is interesting to compare our experiments to those using double charge transfer (DCT). As DCT is a charge-transfer reaction due to collision with a medium-energy ion beam, it is believed to result in a truly vertical transition to the dication [16-18]. On the other hand, an influence of the nuclear dynamics in the core-ionized state on Auger spectra is well known. Its discussion has mostly taken place within the framework of lifetime vibrational interference theory (see e.g., [9,40]). For $\mathrm{CH}_{4}$, the core-ionized state is tetrahedral, with a slight contraction of the $\mathrm{C}-\mathrm{H}$ bonds [34]. It is likely that this direction in nuclear coordinate space corresponds to the repulsive part of the potential-energy curve. We thus expect our technique to result in larger energies for the doubly ionized states than DCT. Figure 4 shows that the values for all states, except that for the $1 t_{2}^{-2}\left({ }^{3} T_{1}\right)$ state, correspond to this expectation; even for the latter, the size of the error bar does not rule out the possibility that our energy is actually larger.

Quantitatively, for the well-resolved states $2 a_{1}^{-1} 1 t_{2}^{-1}\left({ }^{1} T_{2},{ }^{3} T_{2}\right)$ and $1 t_{2}^{-2}\left({ }^{1} A_{1}\right)$ the values obtained by DCT spectroscopy are $1-2.5 \mathrm{eV}$ lower than those from Auger spectroscopy. The DCT process provides the double-ionization energy at the equilibrium distance of the ground state of $\mathrm{CH}_{4}$, which is $\cong 0.05 \AA$ larger than that of the $\mathrm{C} 1 s^{-1}$ core-ionized state [34]. We can further interpret these systematic differences by estimating their size from the slope of the corresponding potential-energy curve, which is another result of our fit algorithm (see Table I). Our results for the slope lie between -20 and $-40 \mathrm{eV} / \AA$ at the equilibrium distance of $1.04 \AA$ for $\mathrm{C} 1 s^{-1}$ ionized $\mathrm{CH}_{4}$. Consequently, $\left[E_{\text {expt }}(\mathrm{Au})-E_{\text {expt }}(\mathrm{DCT})\right] /\left(R_{e, 1 s^{-1}}-R_{e, g s}\right)$ results in -20 to $-50 \mathrm{eV} / \AA$, in good agreement with the slopes derived in the fit analysis. For the states $1 t_{2}^{-2}\left({ }^{1} T_{2},{ }^{1} E,{ }^{3} T_{1}\right)$, the differences of the energy positions obtained by Auger and DCT spectroscopy are less pronounced. However, they are not in contradiction with the slopes due to the large error bars.

The slope of the $1 t_{2}^{-2}\left({ }^{1} A_{1}\right)$ potential-energy curve was also estimated independently based on the theoretical results of Flammini et al. [23]. According to their analysis, the energy of this state in the ground-state geometry is $3.9 \mathrm{eV}$ above a local minimum at an equilibrium distance of $1.32 \AA$. Since this final state also has tetrahedral geometry, we approximated its potential-energy surface along the $\mathrm{C}-\mathrm{H}$ internuclear distance with a harmonic oscillator using $V(R)=\alpha\left(R-R_{e}\right)^{2}$, and in this way we obtained, on the basis of the theoretical results, $\alpha=73.7 \mathrm{eV} / \AA^{2}$. Within this approximation, we obtain a slope of $-41.3 \mathrm{eV} / \AA$ at the equilibrium distance of $1.04 \AA$ for the core-ionized state, in good agreement with the present value of $-37.5(1.6) \mathrm{eV} / \AA$.

Comparison of our results with the various theoretical approaches to $\mathrm{CH}_{4}^{2+}$ leads to a diverse picture without a clear preference for one of them. As mentioned above, the CI calculations of Kwalheim [36] reproduce most of the relative energy differences between states. The same is true for the ADC(2) work of Tarantelli et al. [39], although in absolute energy there is a discrepancy of $2.5 \mathrm{eV}$. In one of the experimental papers, relative state energies were derived from Auger decay, without, however, resolving the Auger spectra due to the different vibrational states [22]. The results of this latter work do not agree with ours, for reasons that are not understood at present.

The present analysis is limited in its accuracy by data statistics. Improved data might allow the $v=2$ Auger spectrum to be extracted. This would provide an additional spectrum that can be included in the analysis and would require only two additional fit parameters for background subtraction. More spectra could be included in the data analysis by measuring $\mathrm{CD}_{4}$. Such a larger data base would probably allow effects to be studied that are beyond the model described above, such as slight curvatures in the potential-energy curves or even possible Jahn-Teller splittings in the degenerate final states.

So far we have not compared our results to two other coincidence methods with which a number of dicationic states of small molecules have been investigated in recent years. However, the coincident detection of photoelectrons from direct photodouble ionization (TOF-PEPECO), as established by Eland, was in the case of methane not able to separate the dicationic states [20]. And the coincident detection of threshold photoelectrons (TPEsCO), which has been applied successfully to small molecules such as $\mathrm{N}_{2}, \mathrm{CO}$, NO, or $\mathrm{O}_{2}$ [41], has not yet been used to study $\mathrm{CH}_{4}$.

In summary, we have been able to separate the Auger spectra corresponding to the $\mathrm{C} 1 s^{-1}(v=0)$ and $\mathrm{C}^{-1} s^{-1}(v=$ 1) vibrational substates of singly core-ionized $\mathrm{CH}_{4}$ using highresolution photoelectron-Auger-electron coincidence spectroscopy. The two spectra show pronounced differences due to the fact that Auger transitions into dissociative states mirror the $\left|\chi_{\text {vib }}(Q)\right|^{2}$ distribution of the particular vibrational substate. By implementing these observations in an analysis in which both spectra are fitted simultaneously, we have been able to separate all seven Auger transitions present in this energy region. We were also able to derive the energy positions and slopes of the potential-energy curves of the $\mathrm{CH}_{4}^{2+}$ final states as well as the relative Auger intensities. There is good agreement with the data obtained from DCT spectroscopy and theoretical results. The combination of photoelectron-Augerelectron coincidence measurements with our approach to data analysis has thus allowed us to obtain detailed information on the energies of dicationic states and their repulsive 
potential-energy curves for bound-dissociative transitions in small molecules.

This approach is of general applicability and can-in principle-be used to derive information on the potentialenergy curves (energy position and slopes) of the dicationic states of a variety of small molecules. The only prerequisite for such an analysis is that vibrational substates are clearly resolved in the photoelectron spectrum. The present experimental setup can be applied, for example, to C $1 s^{-1}$ ionization in $\mathrm{CO}, \mathrm{Si} 2 p^{-1}$ ionization in $\mathrm{SiH}_{4}$ and $\mathrm{SiD}_{4}$, and S $2 p^{-1}$ ionization in OCS. A planned improvement by a factor of approximately 2 in the experimental resolution in the photoelectron channel will make it comparable to the natural linewidth and the vibrational splitting of most shallow-core ionized states. In this way, the method will be applicable to a much larger number of molecular species.

\section{ACKNOWLEDGMENTS}

This work was partially funded by the Deutsche Forschungsgemeinschaft (Contracts No. Pu 180/6-1 and No. He 3060/4-3) and by the Fonds der Chemischen Industrie.
[1] G. A. Sawatzky, Phys. Rev. Lett. 39, 504 (1977).

[2] W. Eberhardt, J. Stöhr, J. Feldhaus, E. W. Plummer, and F. Sette, Phys. Rev. Lett. 51, 2370 (1983).

[3] U. Hergenhahn, J. Phys. B 37, R89 (2004).

[4] H. W. Haak, G. A. Sawatzky, and T. D. Thomas, Phys. Rev. Lett. 41, 1825 (1978).

[5] K. Lee, S. L. Hulbert, P. Kuiper, D. Ji, and D. M. Hanson, Nucl. Instrum. Methods Phys. Res. A 347, 446 (1994).

[6] Y. Hikosaka, P. Lablanquie, F. Penent, J. G. Lambourne, R. I. Hall, T. Aoto, and K. Ito, J. Electron Spectrosc. Relat. Phenom. 137-140, 287 (2004).

[7] P. Bolognesi, M. Coreno, L. Avaldi, L. Storchi, and F. Tarantelli, J. Chem. Phys. 125, 054306 (2006).

[8] P. Bolognesi, P. O’Keeffe, and L. Avaldi, J. Phys. Chem. A 113, 15136 (2009).

[9] P. Bolognesi, R. Püttner, and L. Avaldi, Chem. Phys. Lett. 464, 21 (2008).

[10] V. Ulrich, S. Barth, S. Joshi, T. Lischke, A. M. Bradshaw, and U. Hergenhahn, Phys. Rev. Lett. 100, 143003 (2008).

[11] V. Ulrich, S. Barth, T. Lischke, S. Joshi, T. Arion, M. Mucke, M. Förstel, A. M. Bradshaw, and U. Hergenhahn, J. Electron Spectrosc. Relat. Phenom. 183, 70 (2011).

[12] D. Schröder and H. Schwarz, J. Phys. Chem. A 103, 7385 (1999).

[13] A. Kivimäki, M. Neeb, B. Kempgens, H. M. Köppe, and A. M. Bradshaw, J. Phys. B 29, 2701 (1996).

[14] R. Spohr, T. Bergmark, N. Magnusson, L. O. Werme, C. Nordling, and K. Siegbahn, Phys. Scr. 2, 31 (1970).

[15] K. Nagesha, V. R. Marathe, and D. Mathur, Rapid Commun. Mass Spectrom. 5, 15 (1991).

[16] P. G. Fournier, J. Fournier, F. Salama, P. J. Richardson, and J. H. D. Eland, J. Chem. Phys. 83, 241 (1985).

[17] C. J. Reid, Int. J. Mass Spectrom. Ion Proc. 110, 195 (1991).

[18] W. J. Griffiths, S. Svensson, A. Naves de Brito, N. Correia, M. L. Langford, and F. M. Harris, Rapid Commun. Mass Spectrom. 6, 438 (1992).

[19] P. A. Hatherly, M. Stankiewicz, L. J. Frasinski, K. Codling, and M. A. Macdonald, Chem. Phys. Lett. 159, 355 (1989).

[20] J. H. D. Eland, Chem. Phys. 323, 391 (2006).

[21] E. Kukk, J. Rius Riu, M. Stankiewicz, P. Hatherly, P. Erman, E. Rachlew, P. Winiarczyk, M. Huttula, and S. Aksela, Phys. Rev. A 66, 012704 (2002).
[22] E. Kukk, G. Prümper, R. Sankari, M. Hoshino, C. Makochekanwa, M. Kitajima, H. Tanaka, H. Yoshida, Y. Tamenori, E. Rachlew, and K. Ueda, J. Phys. B 40, 3677 (2007).

[23] R. Flammini, M. Satta, E. Fainelli, G. Alberti, F. Maracci, and L. Avaldi, New J. Phys. 11, 083006 (2009).

[24] P. E. M. Siegbahn, Chem. Phys. 66, 443 (1982).

[25] M. Sironi, D. L. Cooper, J. Gerratt, and M. Raimondi, Mol. Phys. 65, 251 (1988).

[26] M. W. Wong and L. Radom, J. Am. Chem. Soc. 111, 1155 (1989).

[27] E. A. Gislason, J. Chem. Phys. 58, 3702 (1973).

[28] J. Bahrdt, W. Frentrup, A. Gaupp, M. Scheer, W. Gudat, G. Ingold, and S. Sasaki, Nucl. Instrum. Methods Phys. Res. A 467-468, 21 (2001).

[29] F. Senf, F. Eggenstein, R. Follath, S. Hartlaub, H. Lammert, T. Noll, J. S. Schmidt, G. Reichardt, O. Schwarzkopf, M. Weiss, T. Zeschke, and W. Gudat, Nucl. Instrum. Methods Phys. Res. A 467-468, 474 (2001).

[30] O. Kugeler, S. Marburger, and U. Hergenhahn, Rev. Sci. Instrum. 74, 3955 (2003).

[31] V. Myrseth, J. D. Bozek, E. Kukk, L. J. Sæthre, and T. D. Thomas, J. Electron Spectrosc. Relat. Phenom. 122, 57 (2002).

[32] H. M. Köppe, B. S. Itchkawitz, A. L. D. Kilcoyne, J. Feldhaus, B. Kempgens, A. Kivimäki, M. Neeb, and A. M. Bradshaw, Phys. Rev. A 53, 4120 (1996).

[33] T. X. Carroll, N. Berrah, J. Bozek, J. Hahne, E. Kukk, L. J. Sæthre, and T. D. Thomas, Phys. Rev. A 59, 3386 (1999).

[34] T. Karlsen and K. J. Børve, J. Chem. Phys. 112, 7986 (2000).

[35] I. B. Ortenburger and P. S. Bagus, Phys. Rev. A 11, 1501 (1975).

[36] O. M. Kvalheim, Chem. Phys. Lett. 86, 159 (1982).

[37] G. Herzberg, Molecular Spectra and Molecular Structure I. Spectra of Diatomic Molecules (Van Nostrand, New York, 1950), p. 392.

[38] M. Higashi, E. Hiroike, and T. Nakajima, Chem. Phys. 68, 377 (1982).

[39] F. Tarantelli, J. Schirmer, A. Sgamellotti, and L. S. Cederbaum, Chem. Phys. Lett. 122, 169 (1985).

[40] R. Püttner, V. Pennanen, T. Matila, A. Kivimäki, M. Jurvansuu, H. Aksela, and S. Aksela, Phys. Rev. A 65, 042505 (2002), and references therein.

[41] G. Dawber, A. G. McConkey, L. Avaldi, M. A. MacDonald, G. C. King, and R. I. Hall, J. Phys. B 27, 2191 (1994). 